\title{
Physical Gelation of a Bacterial Thermoplastic Elastomer
}

\author{
H. W. Richtering, K. D. Gagnon, R. W. Lenz," R. C. Fuller, and H. H. Winter* \\ Department of Polymer Science \& Engineering, University of Massachusetts, \\ Amherst, Massachusetts 01003
}

Received September 25, 1991; Revised Manuscript Received January 9, 1992

\begin{abstract}
The crystallization of a bacterial polyester, poly ( $\beta$-hydroxyoctanoate), produced by $P$ seudomonas oleovorans was studied by dynamic mechanical measurements. Samples were first preheated to a temperature $T_{\mathrm{p}}$ above the melting temperature, $T_{\mathrm{m}}$, and then supercooled for isothermal crystallization at temperatures of $T_{\mathrm{c}}=15$ or $20^{\circ} \mathrm{C}$. Storage and loss moduli $G^{\prime}(\omega)$ and $G^{\prime \prime}(\omega)$ grew with crystallization time since the crystallites connected the macromolecules into a three-dimensional physical network. The final solid polymer was a thermoplastic elastomer. At the gel point the sample is characterized by the typical self-similar relaxation modulus, $G(t)=S t^{-n}$ with $n=0.11$, and a frequency-independent loss angle. From measurements at different crystallization times a master curve was obtained by time-cure superposition. Such master curves have the same shape for crystallization at $T_{\mathrm{c}}=15$ and $20^{\circ} \mathrm{C}$. The results of the rheological measurements support the suggestion that crystallization-induced physical gelation is characterized by dynamic self-similarity which was observed earlier for chemical gelation. However, when crystallizing at a higher temperature $\left(T_{\mathrm{c}}=30^{\circ} \mathrm{C}\right)$, no critical gel was observed macroscopically which indicated that an inhomogeneous crystallization occurred, leading to a nonuniform gelation process.
\end{abstract}

\section{Introduction}

Bacterial polyesters have attracted widespread interest partly because they are biodegradable. Poly $(\beta$-hydroxyalkanoates) (PHAs) have been known for a long time, and poly( $\beta$-hydroxybutyrate) (PHB), which is highly crystalline, has already found commercial application as a thermoplastic polymer. ${ }^{1,2}$ Depending on the structure of the PHA and the degree of crystallinity, these polymers can behave as thermoplastic elastomers (TPEs). ${ }^{3,4}$ Above the melting temperature, $T_{\mathrm{m}}$, such TPEs can be processed in the liquid state. Below $T_{\mathrm{m}}$ (and above the glass transition temperature, $T_{\mathrm{g}}$ ) TPEs are viscoelastic solids. In this temperature range segments of the macromolecules are able to form crystalline domains which act as physical cross-links for the remaining amorphous chain segments.

The solidification process of such thermoplastic elastomers can be viewed as physical gelation. When the polymer is quenched from the molten state to a temperature $T_{\mathrm{c}}$ below $T_{\mathrm{m}}$ (and above $T_{\mathrm{g}}$ ), the crystalline domains form gradually by means of a nucleation and growth process. These crystalline domains act as physical crosslinks which connect molecules into larger clusters and, vice versa, each of the clusters contains many crystalline domains. The cluster size distribution is assumed to be broad. With an increase in the degree of crystallinity, $p$, the number and size of the clusters increase. The growing crystalline fraction can be observed through the increasing time constants of the viscoelastic relaxation spectrum. At a critical crystalline fraction, $p_{\mathrm{c}}$, the polymer undergoes a transition from a viscoelastic liquid to a viscoelastic solid. This transition point has been termed "gel point", and the polymer at the gel point (GP) is referred to as a "critical gel ${ }^{n}$.

There are many similarities between physical gelation and the more widely studied chemical gelation. The gel point is defined as the instant at which the weight-average molar mass diverges to infinity (infinite sample size) or the largest cluster extends throughout the entire sample (finite sample size). The critical gel has no intrinsic size scale, except for the distance between cross-links, and the static structure is self-similar. There also exists no intrinsic time scale for this structure; i.e., the relaxation modes are self-similar and the relaxation modulus reduces to ${ }^{5,6}$

$$
G(t)=S t^{-n} \quad \text { at GP, for } \lambda_{0}<t<\infty
$$

The gel strength, $S$, and the relaxation exponent, $n$, are characteristic properties of the critical gel and depend on the details of the molecular structure in some unknown way. $\lambda_{0}$ is the lower cutoff of the self-similarity due to the glassy response at small scale. The dynamic mechanical behavior at the gel point is given by a power law relation between moduli and frequency

$$
G^{\prime}(\omega) \propto G^{\prime \prime}(\omega) \propto \omega^{n}, \quad 0<\omega<1 / \lambda_{0}
$$

i.e., storage $\left(G^{\prime}\right)$ and loss moduli $\left(G^{\prime \prime}\right)$ are parallel in a $\log -\log$ plot. Consequently the loss angle is independent of frequency at the gel point

$$
\delta_{\mathrm{c}}=n \pi / 2 \text { or } \tan \delta=G^{\prime \prime} / G^{\prime}=\tan (n \pi / 2)
$$

and $\tan \delta$ data provide a convenient method for determining the gel point.

The relaxation modulus at the gel point has been measured for some physical gels, and the same scaling behavior was found. . $^{-9}$ In physical gels the formation of cross-links is reversible, and the finite lifetime of crosslinks can affect the dynamic behavior of the gel. The main difference between physical and chemical gels lies in the structure of the cross-links. In physical gels, the crosslinking domains can be very large, distributed in size and of high functionality because each cross-link involves many chains, whereas in chemical gels the branching point is well-defined by the functionality of the molecules.

The finite lifetime of the physical junctions introduces an upper cutoff for the self-similar behavior in eq $1, \lambda_{0}<$ $t<\lambda_{\max }$. For crystallizing systems, however, this longest relaxation time is extremely long. It can be considered infinite on the time scale of this study.

Recently Lin and co-workers studied the evolution of the rheological properties during crystallization of a thermoplastic, elastomeric polypropylene with linear macromolecules which consisted of statistical sequences of atactic and stereoregular blocks. ${ }^{9}$ A strong influence of the thermal history on the gelation process was observed, and, depending on the preheating temperature, scaling behavior was found in some cases. 


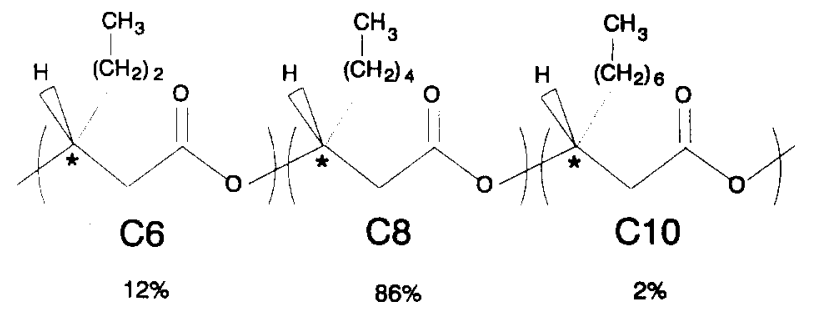

Figure 1. Chemical structure of PHO.

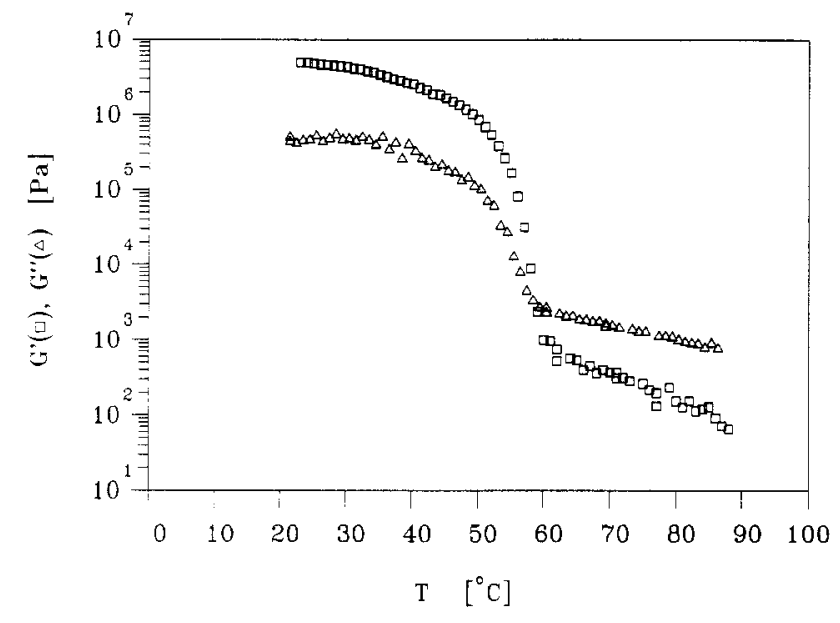

Figure 2. Melting transition of $\mathrm{PHO}$ at $\omega=1 \mathrm{rad} / \mathrm{s}$. The strain amplitude was $0.2-10 \%$.

In this study, we investigated poly ( $\beta$-hydroxyoctanoate) (PHO), a polyester produced by $P$ seudomonas oleovorans when grown with sodium octanoate as the sole carbon substrate. Even though PHO is obtained from a single substrate, it is a copolymer; the structure is shown in Figure 1. ${ }^{10,11}$ The numbers in Figure 1 indicate the composition in mole percent. The different repeating units are distributed randomly along the chains.

The crystallinity of $\mathrm{PHO}$ is approximately $25 \%$ in weight. $^{3,10,11}$ It shows elastic properties, while PHB is a rigid brittle plastic because of its high degree of crystallinity and a relatively high $T_{\mathrm{g}}{ }^{12}$ The mechanical properties of PHO like tensile strength, modulus, and hardness are in the range of commercially available TPEs. ${ }^{4}$

The purpose of this study is to further examine whether crystallization-induced gelation follows the same dynamic behavior as chemical gelation. Further understanding of the crystallization will help in the search for suitable bacterial polyesters.

\section{Experimental Section}

The bacterial production and characterization of PHO was previously described. ${ }^{10,11}$ A Rheometrics mechanical spectrometer (RMS-800) and a dynamic spectrometer (RDS Model 7700) with a parallel-plate geometry were used for dynamic mechanical measurements. The rheological data were analyzed with the standard IRIS software. DSC measurements were performed with a Du Pont differential scanning calorimeter Model 2910 at a heating rate of $20 \mathrm{~K} / \mathrm{min}$.

\section{Results}

The DSC thermogram of PHO shows a glass transition at $-35^{\circ} \mathrm{C}$ and a melting transition at approximately $55^{\circ} \mathrm{C}$ depending on the crystallization temperature. When $\mathrm{PHO}$ was gradually heated, the storage $\left(G^{\prime}\right)$ and loss $\left(G^{\prime \prime}\right)$ moduli at $1 \mathrm{rad} / \mathrm{s}$ decayed by several orders of magnitude during the melting transition at about $55^{\circ} \mathrm{C}$ as shown in Figure 2.

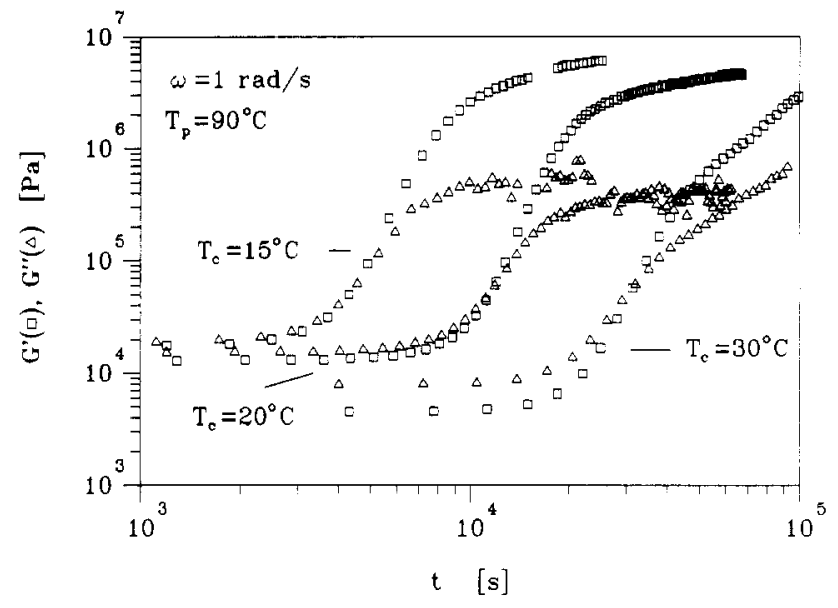

Figure 3. Growth of moduli as a function of crystallization time at three different temperatures. $T_{\mathrm{p}}=90^{\circ} \mathrm{C}$.

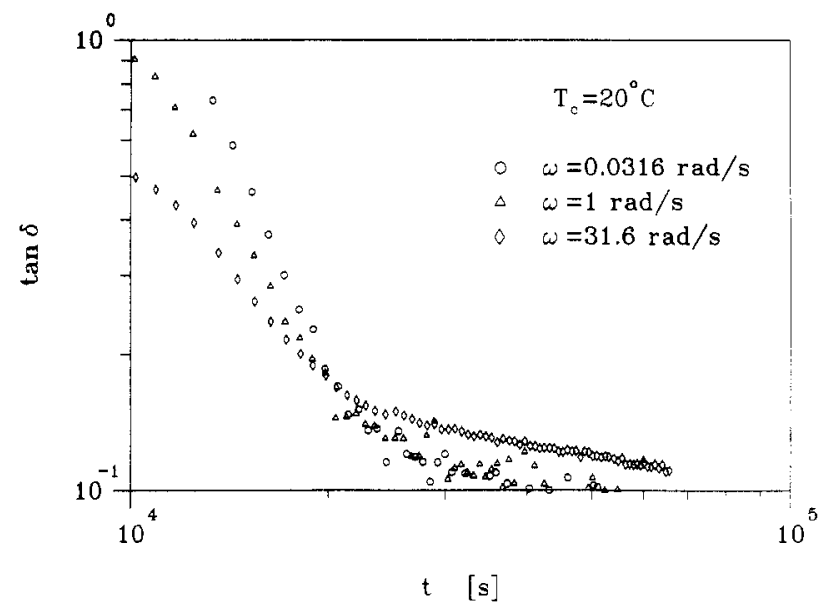

Figure 4. Crystallization time dependence of $\tan \delta$ for three different frequencies. $T_{\mathrm{c}}=20^{\circ} \mathrm{C}$.

In order to study crystallization, samples of PHO were first melted at a preheating temperature, $T_{\mathrm{p}}=90$ or 70 ${ }^{\circ} \mathrm{C}$. At time $t=0$, the samples were quenched to a temperature $T_{\mathrm{c}}$ and held there for isothermal crystallization. The dynamic moduli at $1 \mathrm{rad} / \mathrm{s}$ increased with crystallization time as shown in Figure 3. Crystallization temperatures, $T_{\mathrm{c}}=15,20,30^{\circ} \mathrm{C}$, were appropriate for the polymer for two reasons. First, no influence of vitrification on the dynamic properties is expected at temperatures of $50 \mathrm{~K}$ or more above $T_{\mathrm{g}}$. Second, the crystallization rate is so slow that the properties of the sample do not change significantly during individual $G^{\prime}$ and $G^{\prime \prime}$ measurements. At all temperatures a long nucleation time was observed. Data taken at $T_{\mathrm{p}}=70^{\circ} \mathrm{C}$ were indistinguishable from the $T_{\mathrm{p}}=90^{\circ} \mathrm{C}$ data, and therefore only the latter are shown in Figure 3.

According to eq 3 the critical gel is characterized by a loss tangent which is independent of frequency. Therefore, $\tan \delta$ was determined at different frequencies as a function of crystallization time. For $T_{\mathrm{c}}=20^{\circ} \mathrm{C}$ and $T_{\mathrm{p}}=90^{\circ} \mathrm{C}$, the gel point (GP) was reached after $2 \times 10^{4} \mathrm{~s}(5.5 \mathrm{~h})$, as seen in Figure 4. Before GP, $\tan \delta$ decreased with increasing frequency (Figure 5), as is typical for a viscoelastic liquid. After GP, $\tan \delta$ increased with frequency, as is typical for a viscoelastic solid; the sample had changed from a viscoelastic liquid to a viscoelastic solid. At GP, $\tan \delta=0.17$ corresponded to $n=0.11$, which is slightly smaller than that for the thermoplastic elastomeric polypropylene. ${ }^{9}$ The prefactor is $S=1.39 \times 10^{6} \mathrm{~Pa} \cdot \mathrm{s}^{n}$. The behavior of the storage and loss moduli is shown in Figure 


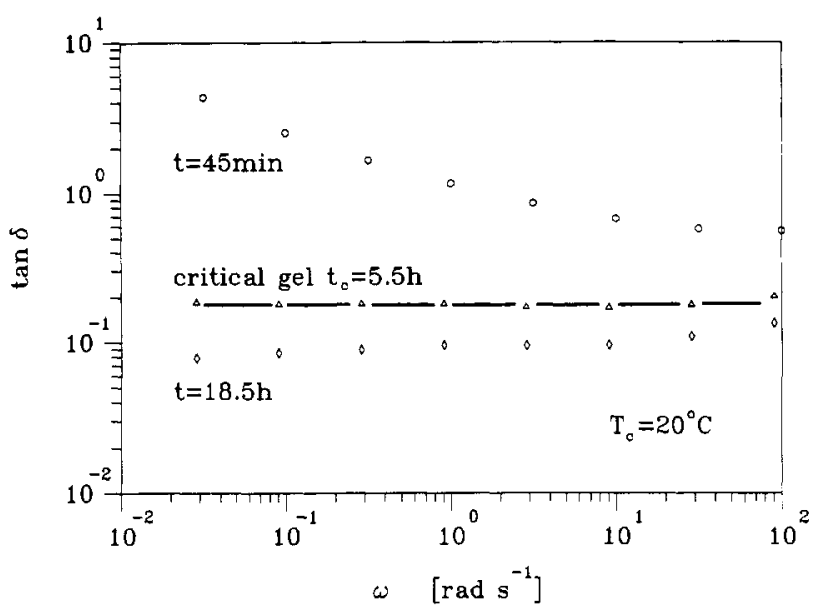

Figure 5. Frequency dependence of $\tan \delta$ at three different times. $T_{\mathrm{c}}=20^{\circ} \mathrm{C}$.

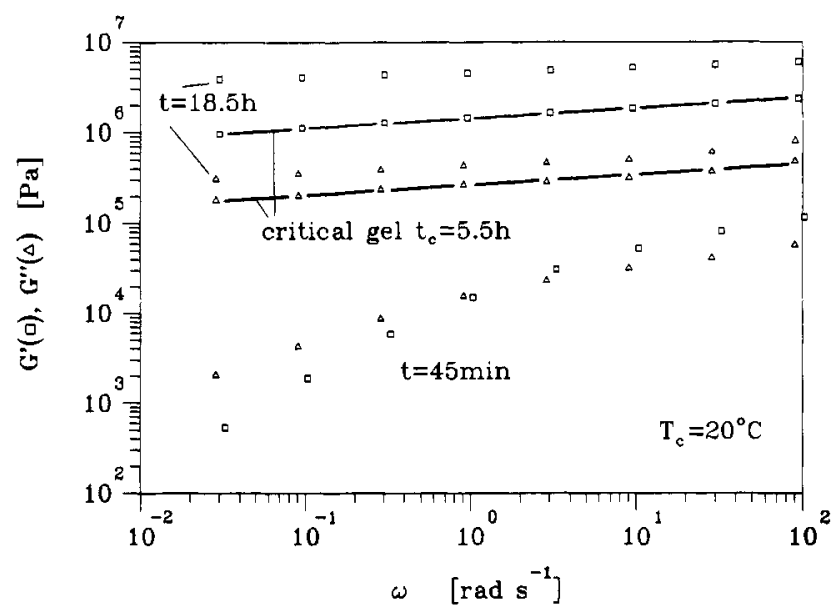

Figure 6. Frequency dependence of $G^{\prime}$ and $G^{\prime \prime}$ at three different times. $T_{c}=20^{\circ} \mathrm{C}$.

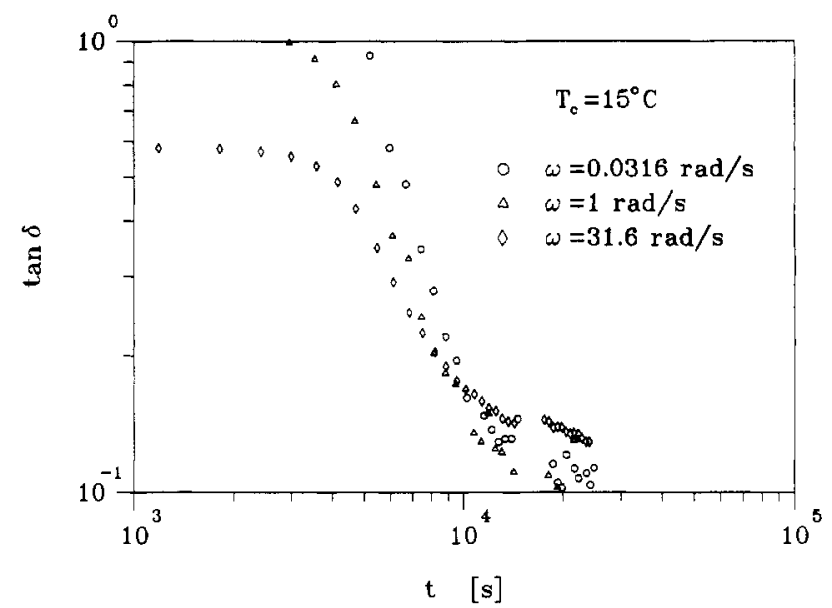

Figure 7. Crystallization time dependence of $\tan \delta$ for three different frequencies. $T_{c}=15^{\circ} \mathrm{C}$.

6. As expected, $G^{\prime}(\omega)$ and $G^{\prime \prime}(\omega)$ are parallel to each other at the gel point.

The influence of the crystallization temperature on the developing structure can be seen in Figures 7 and 8 . At $T_{\mathrm{c}}=15^{\circ} \mathrm{C}$ crystallization is much faster and $\mathrm{GP}$ is reached after $10^{4} \mathrm{~s}$. The critical exponent has about the same value as obtained at $20^{\circ} \mathrm{C}$ while the prefactor is higher, $S=2.5$ $\times 10^{6} \mathrm{Pa \cdot \textrm {s } ^ { n }}$. At $T_{\mathrm{c}}=30^{\circ} \mathrm{C}$, however, the crystallization behavior is significantly different. $\tan \delta$ curves measured at different frequencies do not cross each other at one specific time, and at no time during the gelation process

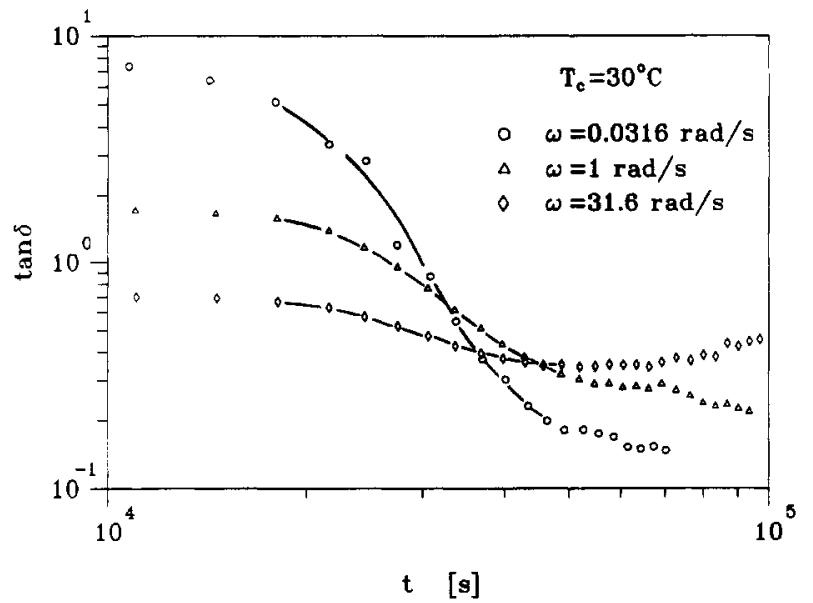

Figure 8. Crystallization time dependence of $\tan \delta$ for three different frequencies. $T_{\mathrm{c}}=30^{\circ} \mathrm{C}$.

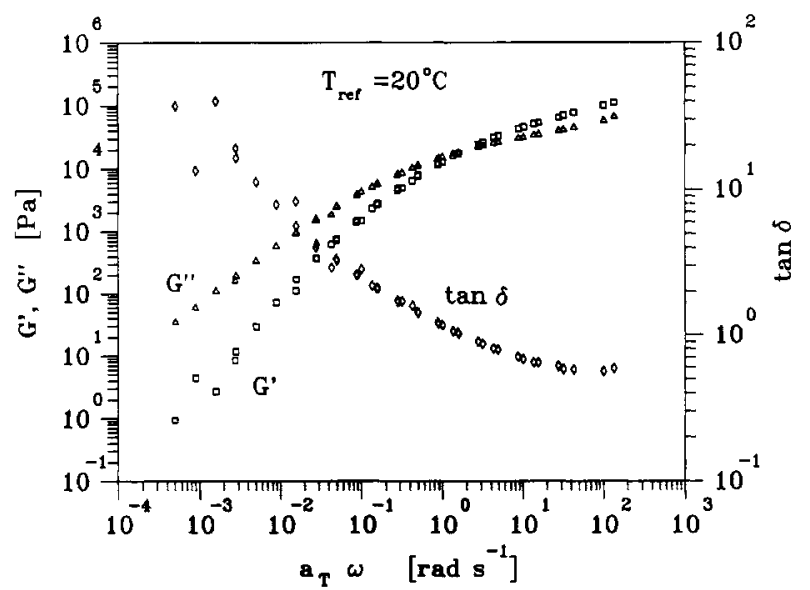

Figure 9. Master curve from time-temperature superposition before the onset of crystallization.

is $\tan \delta$ independent of frequency; that is, no critical gel was observed in the macroscopic rheological experiment.

\section{Discussion}

The crystallization process of PHO apparently depends strongly on $T_{\mathrm{c}}$. From Figure 3 it can be seen that the crystallization of $\mathrm{PHO}$ involves a long lag time. Thus, the first frequency sweeps probe the amorphous melt in a supercooled state. These measurements can be used to extend the frequency range of the $G^{\prime}$ and $G^{\prime \prime}$ master curves in the liquid state by applying the time-temperature superposition principle; see Figure 9 . Before crystallization the behavior is typical for a polymeric liquid.13 In Figure 3 the moduli at all three temperatures are plotted at the same frequency $(\omega=1 \mathrm{rad} / \mathrm{s})$. If instead the shifted frequency $a_{\mathrm{T}} \omega$ is used, all curves start with the same value of $G^{\prime}$ and $G^{\prime \prime}$, respectively.

The evolution of $G^{\prime}$ and $G^{\prime \prime}$ (Figure 3) and of $\tan \delta$ (Figures 4, 7, and 8) indicates that different crystallization processes occurred at different extents of supercooling. The results will be discussed first for $T_{\mathrm{c}}=15$ and $20^{\circ} \mathrm{C}$, where the evolution of rheology shows the same characteristic features as is known for chemical gelation: at a specific time during the gelation process $G^{\prime}$ and $G^{\prime \prime}$ follow a power law; i.e., a critical gel has been formed. Apparently, differences in cross-link structure between the thermoplastic elastomer, $\mathrm{PHO}$, and known chemically cross-linked samples do not alter the behavior at the gel point. Molecular clusters with a self-similar structure are present in the critical gel. The values of the gel strength and the 


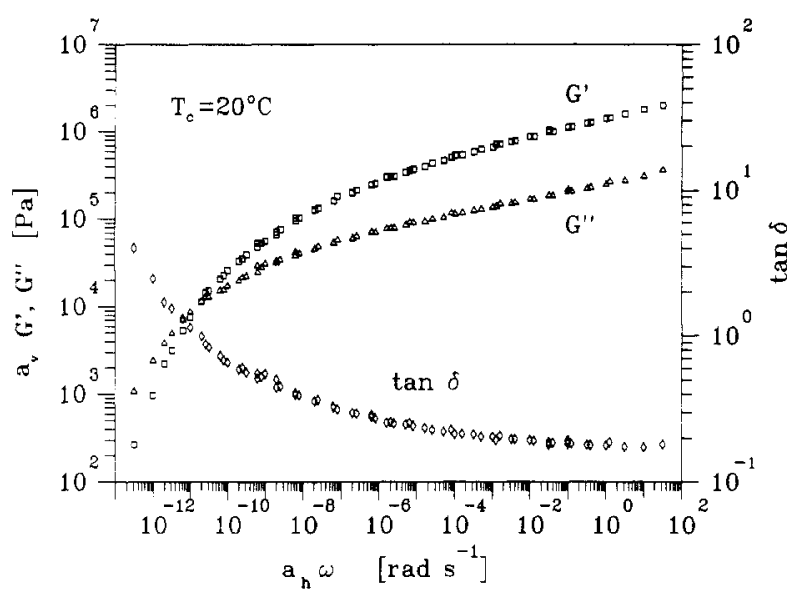

Figure 10. Master curve from time-cure superposition for crytallization at $T_{\mathrm{c}}=20^{\circ} \mathrm{C}$.

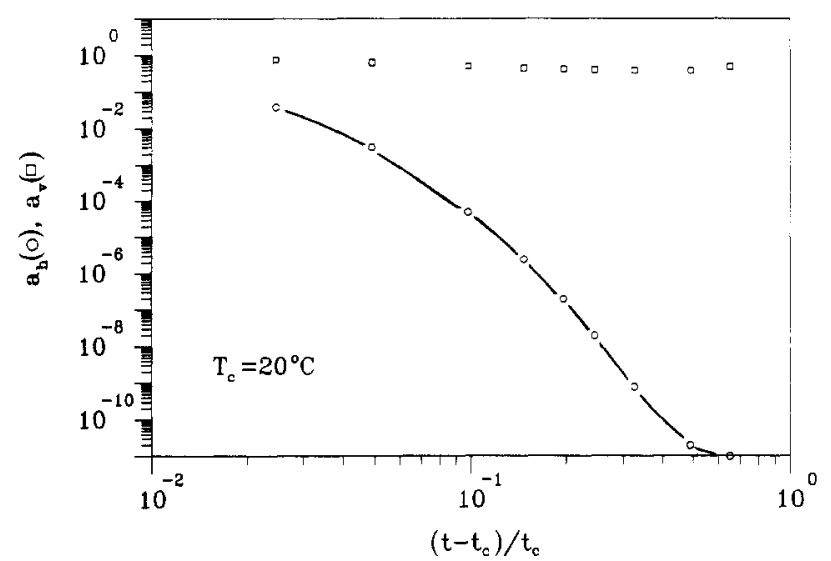

Figure 11. Horizontal $\left(a_{\mathrm{h}}\right)$ and vertical $\left(a_{\mathrm{v}}\right)$ shift factors of timecure shifts vs the distance to the critical time for crystallization at $T_{\mathrm{c}}=20^{\circ} \mathrm{C}$.

relaxation exponent are similar to the values found for polypropylene. In both systems the gel strength is higher compared to poly(dimethylsiloxane) networks. ${ }^{14}$ The relaxation exponent is very low, which seems to be characteristic for cross-linking molecules with high molecular weight.

From theoretical considerations it was suggested that the viscoelastic functions at different extents of network formation may be superposed if appropriate horizontal and vertical shifts are applied. ${ }^{15,16}$ In other words, master curves can be obtained by time-cure superposition, if snapshots of $G^{\prime}(\omega)$ and $G^{\prime \prime}(\omega)$ at discrete times are shifted appropriately. It was shown experimentally that such master curves can be constructed in the case of chemical gels. ${ }^{17,18}$ Figure 10 shows that this procedure is also valid for crystallization-induced physical gelation. $G^{\prime}(\omega)$ and $G^{\prime \prime}(\omega)$ at 10 discrete times $t \leq t_{\mathrm{c}}\left(t_{\mathrm{c}}\right.$ denotes the time when the critical gel is observed) could be shifted into a single set of master curves as shown in Figure 10. In Figure 11 the cure shift factors are plotted against the distance from the critical gel time. Time-cure superposition should be possible both before and after the critical gel. In the case of PHO, however, time-cure shifting after the gel point was not possible, because the frequency dependence of $\tan \delta$ was very weak and shifting became ambiguous.

A master curve can also be obtained for crystallization of $\mathrm{PHO}$ at $15^{\circ} \mathrm{C}$. The critical gels at 15 and $20^{\circ} \mathrm{C}$ have the same relaxation exponent $n$, and the time-curve master curves are of the same shape and they can be superposed by an additional temperature shift. The observation that time-temperature superposition can be applied to the

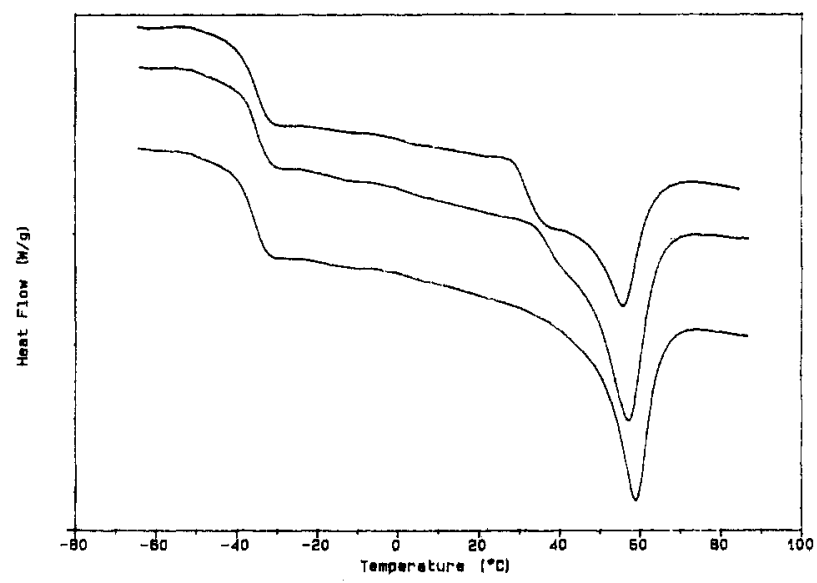

Figure 12. DSC diagrams obtained after crystallization for 24 $\mathrm{h}$ at 20,30 , and $35^{\circ} \mathrm{C}$ (from top to bottom).

crystallization process of $\mathrm{PHO}$ at 15 and $20^{\circ} \mathrm{C}$ indicates that the gel structure is basically the same at both temperatures.

When crystallizing at $T_{\mathrm{c}}=30^{\circ} \mathrm{C}$, no critical gel was observed. Apparently the sample changed from a viscoelastic liquid to a viscoelastic solid without going through an intermediate stage which obeys the self-similar structure of a critical gel. Obviously the network structure depends on the crystallization temperature. A possible explanation for the different gelation behavior can be given by different crystallization kinetics. When crystallizing at low temperatures (i.e., a high degree of supercooling), many nuclei are formed and the critical size of the crystallites is small. However, when crystallizing at a higher temperature, fewer nuclei are formed and the crystallites are larger and have. a higher melting temperature. In Figure 12 DSC thermograms are shown which were obtained after crystallization for $24 \mathrm{~h}$ at 20,30 , and $35^{\circ} \mathrm{C}$, respectively. A different melting temperature at the peak maximum and $a$ different shape of the peak are observed, indicating a different size distribution of the crystallites. The different nucleation and growth kinetics can affect the gelation process. Usually it is assumed that the kinetics of network formation is uniform throughout the entire sample. Slow nucleation, however, can lead to long-range heterogeneity of crystallization rate within the sample. In such a case, the ideal scaling behavior is not expected.

The accessible frequency window of the rheological experiment has been limited by experimental constraints. The behavior at very low frequency has not been probed, and the possibility for self-similar behavior at such very low frequencies should not be excluded. It would mean that only the very large clusters are self-similar in their behavior for these $T_{\mathrm{c}}=30^{\circ} \mathrm{C}$ gels.

A different explanation for deviations from the ideal behavior was recently discussed by Lin and co-workers. ${ }^{9}$ They studied the crystallization of polypropylene and investigated the influence of preheating temperature. When samples were melted at a high $T_{\mathrm{p}}$, a critical gel was observed, whereas at a low $T_{\mathrm{p}}$, no critical gel was found. In this system only the stereoregular blocks were able to crystallize, and microphase-separated structures persisted in the molten state if $T_{\mathrm{p}}$ was not sufficiently high, leading to a nonuniform gelation process if the sample was quenched. Rheological experiments integrate the response of the entire sample, and, therefore, long-range structural fluctuations will lead to deviations from the ideal scaling behavior.

In the case of $\mathrm{PHO}$, the preheating temperature seems not to be as crucial. Measurements at $T_{\mathrm{c}}=30^{\circ} \mathrm{C}$ with $T_{\mathrm{p}}$ 
$=90$ and $70^{\circ} \mathrm{C}$ showed the same dynamic mechanical behavior. However, incompatibilities of different repeating units of $\mathrm{PHO}$ with the crystal structure could influence the crystallization process and, hence, the cluster mass distribution in the gel. Recently Marchessault and coworkers studied the crystal structure of $\mathrm{PHO}$ at room temperature. ${ }^{3}$ From their calculations two structures of similar energy were suggested, but it was not possible to determine which structure was present, and it is still not clear whether or not isomorphism can occur with the repeating units of PHO. Further investigations on the crystallization of PHO are in progress and will be reported later.

\section{Conclusions}

At 15 and $20^{\circ} \mathrm{C}$ the crystallization-induced gelation of the thermoplastic elastomer $\mathrm{PHO}$ revealed the same rheological behavior as is known for chemical gelation. The critical gel was characterized by a power law relaxation spectrum, and the gel point could be determined by dynamic mechanical measurements. Time-cure master curves could be obtained. The observation of a scaling behavior in physical gelation further supports that dynamic self-similarity at the gel point could be a universal phenomenon for all cross-linking systems. The different structure of the cross-linking zones in thermoplastic elastomers compared to chemical gels obviously does not affect the ideal scaling behavior.

At $T_{\mathrm{c}}=30^{\circ} \mathrm{C}$ no critical gel was observed macroscopically, but $\tan \delta$ depended on the frequency at all times during the crystallization process. The absence of a critical gel may be explained by the occurrence of a nonuniform gelation process, indicating that, depending on temperature, crystallization processes do not occur simultaneously but on a time scale which is distributed throughout the sample.
Acknowledgment. H.W.R. cordially thanks the Alexander von Humboldt-Stiftung for a Feodor Lynen Research Fellowship during the period of which this work was performed. Financial support of the Office of Naval Research, Grant No. N00014-84K-0369, is gratefully acknowledged. We also thank Marian Mours for letting us use the GELPRO software for data evaluation of transient samples.

\section{References and Notes}

(1) Holmes, P. A. Phys. Technol. 1985, 16, 32.

(2) Dawes, E. A. Biosci. Rep. 1988, 8, 537.

(3) Marchessault, R. H.; Monasterios, C. J.; Morin, F. G.; Sundararajan, P. R. Int. J. Biol. Macromol. 1990, 12, 158.

(4) Gagnon, K. Ph.D. Thesis, in preparation.

(5) Chambon, F.; Winter, H. H. Polym. Bull. 1985, 13, 499.

(6) Winter, H. H.; Chambon, F. J. Rheol. 1986, 30, 367; 1987, 31, 683.

(7) Te Nijenhuis, K.; Winter, H. H. Macromolecules 1989, 22,411

(8) Cuvelier, G.; Launay, B. Makromol. Chem., Macromol. Symp. $1990,40,23$.

(9) Lin, Y. G.; Mallin, D. T.; Chien, J. C. W.; Winter, H. H. Macromolecules 1991, 24, 850.

(10) Brandl, H.; Gross, R. A.; Lenz, R. W.; Fuller, R. C. Appl. Environ. Microbiol. 1988, 54, 1977.

(11) Gross, R. A.; DeMello, C.; Lenz, R. W.; Brandl, H.; Fuller, R. C. Macromolecules 1989, 22, 1106.

(12) Bauer, H.; Owen, A. T. Colloid Polym. Sci. 1988, 266, 241.

(13) Ferry, J. D. Viscoelastic Properties of Polymers; Wiley: New York, 1980.

(14) Scanlan, J. C.; Winter, H. H. Macromolecules 1991, 24, 47.

(15) Martin, J. E.; Adolf, D.; Wilcoxon, J. P. Phys. Rev. Lett. 1988, $61,2620$.

(16) Martin, J. E.; Adolf, D.; Wilcoxon, J. P. Phys. Rev. A 1989, 39, 1325.

(17) Adolf, D.; Martin, J. E.; Wilcoxon, J. P. Macromolecules 1990, 23,527 .

(18) Hodgson, D. F.; Amis, E. J. Macromolecules 1990, 23, 2512.

Registry No. (C10)(C8)(C6) (copolymer), 128971-75-5; $\mathrm{CH}_{3}\left(\mathrm{CH}_{2}\right)_{6} \mathrm{CO}_{2} \mathrm{H} \cdot \mathrm{Na}, 1984-06-1$. 